(с Е.Б. Башнина*, И.М. Царгасова, О.А. Клиценко

Северо-Западный государственный медицинский университет им. И.И. Мечникова, Санкт-Петербург

ОБОСНОВАНИЕ. Инсулиновые помпы (ИП) получили широкое распространение в лечении сахарного диабета 1 типа (СД1) в детском возрасте в связи с рядом преимуществ по отношению к режиму множественных инъекций инсулина (МИИ). Однако вопросы долгосрочной эффективности непрерывной подкожной инфузии инсулина (НПИИ) в достижении и поддержании стойкой метаболической компенсации по-прежнему не решены.

ЦЕЛЬ. Определить факторы, влияющие на долгосрочную эффективность НПИИ у детей и подростков с СД1 в условиях реальной клинической практики.

МЕтоДЫ. При формировании регистра больных, получающих помповую инсулинотерапию в Санкт-Петербурге, изучены данные 239 детей и подростков, которые находились на НПИИ 3 года и более. Проведен анализ динамики $\mathrm{HbA}_{1 c}$ до перехода на НПИИ и его последнего значения, оценены изменения НbA пациентов, исходной концентрации $\mathrm{HbA}_{1 c^{\prime}}$ а также таких факторов, как частота использования непрерывного мониторирования глюкозы (НМГ), временные переходы с НПИИ на МИИ шприц-ручками, использование калькулятора болюса (КБ).

РЕЗУЛЬТАТЫ. Полученные данные последнего значения $\mathrm{HbA}_{1 c}$ не имеют статистически значимых изменений в срав-

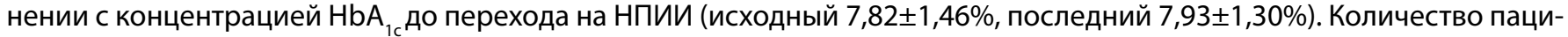
ентов с уровнем $\mathrm{HbA}_{1 c}<7,5 \%$ составляло 42\%. Лучшие показатели наблюдались в группе 4,5-7 лет, где количество пациентов с $\mathrm{HbA}_{1 c}<7,5 \%$ составляло 67\%; в группе 12-18 лет лишь 35\% человек имели целевой НbА пациентов с исходным $\mathrm{HbA}_{1 c}<7,5 \%$ его последнее значение оставалось целевым, в то время как у пациентов с НbА $\geq 7,5 \%$ до перехода на НПИИ лишь $23 \%$ достигли целевого уровня. Также отмечен лучший гликемический контроль у пациентов, которые использовали НПИИ постоянно, в сравнении с пациентами, которые периодически переходили на МИИ с помощью шприц-ручки (р<0,05). НbА НМГ постоянно, по сравнении с группой, в которой не использовали НМГ $(p<0,05)$.

ЗАКЛючЕНИЕ. Применение у детей и подростков с СД1 НПИИ длительностью 3 года и более не привело к значимому улучшению метаболического контроля. Однако прослеживалось влияние на поддержание метаболического контроля таких факторов, как возраст пациентов, исходный НbА вания, а также наличие или отсутствие смен режимов инсулинотерапии.

КЛЮЧЕВЫЕ СЛОВА: дети; подростки; сахарный диабет 1 типа; инсулиновая помпа; гликемия

\title{
SYSTEMS OF CONTINUOUS SUBCUTANEOUS INSULIN INFUSION TO MAINTAIN METABOLIC COMPENSATION FOR TYPE 1 DIABETES MELLITUS AMONG CHILDREN AND ADOLESCENTS
}

(c) Elena B. Bashnina*, Irina M. Tsargasova, Olga A. Klitsenko

North-Western State Medical University named after I.I. Mechnikov, St. Petersburg, Russia

BACKGROUND: Insulin pumps (IP) are a widespread treatment for type 1 diabetes mellitus (T1D) in children because of its several advantages over multiple insulin injection regimens (MII). However, the long-term effectiveness of continuous subcutaneous insulin infusion (CSII) in achieving and maintaining sustained metabolic compensation remains controversial.

OBJECTIVE: To determine the factors affecting the long-team effectiveness of CSII in children and adolescents with T1DM in real clinical practice.

METHODS: Data of 239 children and adolescents treated with CSII for $\geq 3$ years were examined during the registration of patients receiving insulin pump therapy in Saint-Petersburg was formed. $\mathrm{HbA}_{1 c}$ level changes over time were analysed before switching to CSII and were assessed depending on sex and age, baseline $\mathrm{HbA}_{1 \mathrm{c}}$ level and factors as the frequency of using continuous glucose monitoring (CGM), temporary transitions from CSII to MII with injection pens and use of bolus calculators (BC). 
RESULTS: The final $\mathrm{HbA}_{1 \mathrm{c}}$ value showed no significant changes as compared with the $\mathrm{HbA}_{1 \mathrm{c}}$ level before switching to CSII (baseline, 7.82 $\pm 1.46 \%$; final, $7.93 \pm 1.30 \%$ ). Approximately $42 \%$ of patients had $\mathrm{HbA}_{1 c}$ level of $<7.5 \%$. Better results were observed in the 4.5-7-year age group, where $67 \%$ of patients had $\mathrm{HbA}_{1 c}$ level of $<7.5 \%$; in $12-18$-year age group, only $35 \%$ of patients reached the target $\mathrm{HbA}_{1 c}$ level. Majority of patients with baseline $\mathrm{HbA}_{1 \mathrm{c}}$ level of $<7.5 \%$ remained within the target level during the final examination, whereas only $23 \%$ of patients with $\mathrm{HbA}_{1 \mathrm{c}}$ level of $\geq 7.5 \%$ before switching to CSII reached the target level. Better glycaemic control was also observed in patients treated with CSII regularly, as compared to patients who periodically switched to MII with the injection pen $(p<0.05)$. $\mathrm{HbA}_{1 \mathrm{c}}$ appeared to be lower in the group of 12-18-year-old adolescents who used CGM regularly, as compared to the group not treated with CGM ( $p<0.05)$.

CONCLUSION: Children and adolescents treated with CSII for $\geq 3$ years did not experience a significant improvement in metabolic control. However, the influence of factors in maintaining the metabolic control, such factors as the patient's age, initial $\mathrm{HbA}_{1 c}$ level, CGM use and frequency, and the presence or absence of changes in insulin therapy regimen is observed.

KEYWORDS: children; adolescents; type 1 diabetes mellitus; insulin pump; glycemia

Непрерывная подкожная инфузия инсулина (НПИИ), или введение инсулина с помощью инсулиновой помпы (ИП), в настоящее время получила достаточно широкое распространение. Являясь альтернативой режиму множественных инъекций инсулина (МИИ) с помощью шприц-ручки, НПИИ обладает рядом преимуществ:

- возможность применять наиболее физиологические схемы инсулинотерапии путем изменения скорости введения инсулина, что снижает риск гипогликемических состояний;

- смена многократных инъекций инсулина на однократную в течение трех дней значительно повышает уровень качества жизни пациента и его семьи.

Выбор способа введения инсулина в управлении сахарного диабета (СД) в настоящее время является неотъемлемой частью лечебного алгоритма. Вместе с тем успех любого нового вида терапии определяется адекватно выбранными показаниями к данному способу лечения, а также приверженностью и мотивацией пациентов и их родителей [1]. С момента начала НПИИ с помощью ИП в 1970-х гг. популярность помповой инсулинотерапии продолжает расти, и особое место этот способ введения инсулина занимает у подростков [2]. Достижение целевых значений $\mathrm{HbA}_{1 с}$ снижает риск развития острых и хронических осложнений СД $[3,4]$. В большинстве исследований отмечается снижение концентрации $\mathrm{HbA}_{1 с}$ у пациентов на помповой инсулинотерапии, хотя некоторые источники сообщают об отсутствии улучшений или начальном улучшении с последующим возвратом к содержанию $\mathrm{HbA}_{1 с}$ до использования ИП [5-7]. Однако большинство исследований были непродолжительными. Есть данные, указывающие на то, что использование дополнительных технических функций в помпе ассоциировано со значительным улучшением показателя $\mathrm{HbA}_{1 c^{\prime}}$ а лучшие показатели $\mathrm{HbA}_{1 с}$ были обнаружены у мальчиков [7-9]. Большинство современных ИП имеют функцию автоматического расчета доз болюсного инсулина - калькулятор болюса (КБ). Также есть данные, указывающие на то, что использование функции КБ связано с лучшим гликемическим контролем [10].

\section{ЦЕЛЬ}

Оценить долгосрочную эффективность гликемического контроля у детей и подростков с СД 1 типа (СД1), получающих терапию методом НПИИ от от 3 лет и более, в реальной клинической практике, а также определить факторы, влияющие на метаболический контроль.

\section{МАТЕРИАЛ И МЕТОДЫ}

\section{Дизайн исследования}

Проведено ретроспективное обсервационное одноцентровое неконтролируемое исследование.

\section{Критерии соответствия}

В исследование были включены дети и подростки в возрасте от 4,5 до 18 лет с подтвержденным диагнозом СД1, находящиеся на НПИИ от 3 лет и более.

\section{Условия и продолжительность исследования}

Представлены данные о пациентах с СД1, которые находились на НПИИ от 3 лет и более до 01.06.2018. Сбор данных осуществлялся при формировании регистра пациентов, получающих помповую инсулинотерапию, в г. Санкт-Петербурге. Сбор данных о пациентах осуществлялся с 01.05.2018 по 01.06.2018. Анализ данных проводился с 10.09.2018 по 02.02.2019 на базе кафедры эндокринологии им. акад. В.Г. Баранова ФГБОУ ВО «СЗГМУ им. И.И. Мечникова».

\section{Описание медицинского вмешательства}

Данные о пациентах были получены в городском эндокринологическом центре для детей и подростков при формировании регистра пациентов с СД1, получающих НППИ.

\section{Основной исход исследования}

Проводилась оценка изменений последнего значения $\mathrm{HbA}_{1 c}$ в сравнении с исходным как в общей группе пациентов, так и в группах, разделенных по полу, возрасту. Также оценивалось влияние на показатель $\mathrm{HbA}_{1 c}$ таких факторов, как частота использования НМГ, временные переходы с НПИИ на МИИ шприц-ручками, использование КБ. Проводилась оценка изменения последнего значения $\mathrm{HbA}_{1 с}$ по сравнению с исходным, в зависимости от значения $\mathrm{HbA}_{1 c}$ до перехода на НПИИ (<7,5\%, $\left.\geq 7,5 \%\right)$.

\section{Дополнительные исходы исследования}

Дополнительно был проведен письменный опрос, в котором пациенты ответили на вопрос о том, по каким 
причинам они временно переходят с НПИИ на шприцручки, а также причины отказа от НМГ.

\section{Анализ в подгруппах}

Для оценки эффективности терапии и влияния изучаемых факторов на показатель $\mathrm{HbA}_{1 c}$ общая группа условно разделена на подгруппы.

1. По возрасту: 4,5-7 лет, 8-11 лет,12-18 лет.

2. В зависимости от содержания $\mathrm{HbA}_{1 c}$ до перехода на НПИИ (значение 7,5\% использовано как целевое, в соответствии с рекомендациями ISPAD 2014):

- $<7,5 \%$;

- $\geq 7,5 \%$.

3. В зависимости от смены режима инсулинотерапии группа пациентов разделена на 2 подгруппы:

- группа, в которой пациенты используют НПИИ постоянно;

- группа, в которой пациенты временно переходят на МИИ шприц-ручками.

4. В зависимости от частоты использования НМГ:

- не реже 1 нед в месяц;

- не используется, используется 1-2 раза в год, при госпитализациях.

5. В зависимости от использования КБ группа разделена на 2 подгруппы:

- используют;

- не используют.

\section{Этическая экспертиза}

Протокол исследования одобрен локальным этическим комитетом ФГБОУ ВО «СЗГМУ им. И.И. Мечникова», выписка из протокола №4 от 04.04.2018 г. Пациенты дали добровольное согласие на использование их данных в научных целях.

\section{Статистический анализ}

Для анализа полученных в процессе выполнения работы клинических данных использовалась система STATISTICA for Windows (версия 10 Лиц. BXXR310F964808FA-V). С учетом особенностей анализируемых переменных, а также в соответствии с целями и задачами работы выполнены: расчет частотных таблиц (абсолютные значения и проценты в соответствующих группах и подгруппах) как одномерных, так и многоуровневых; расчет элементарных статистик (средние значения, ошибки средних, среднеквадратические отклонения, размах разброса данных, медианы и квартили). Проверку распределения показателей на нормальность выполняли с помощью теста Колмогорова-Смирнова. Анализ частотных характеристик качественных показателей проводился методом $\mathrm{X}^{2}$. Сравнение $\mathrm{HbA}_{1 с}$ в исследуемых группах осуществлялось с использованием критерия Манна-Уитни. Динамика $\mathrm{HbA}_{1 c}$ оценивалась с помощью критерия знаков. Критерием статистической достоверности получаемых выводов считалась величина $\mathrm{p}<0,05$.

\section{РЕЗУЛЬТАТЬ}

Участники исследования

Общая группа включала 239 детей и подростков с СД1 на НПИИ 3 года и более, из них 126 (53\%) де- вочек и 113 (47\%) мальчиков. Средний возраст де-

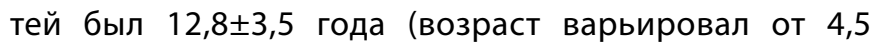
до 18,0 лет), из них 65\% составляли подростки. Средняя продолжительность заболевания - 5,2 23,3 лет. Средняя продолжительность использования НПИИ 5,3 1,8 лет (диапазон от 3 до 12 лет). Использовались ИП Medtronic 712 и 722, Accu Chek Combo с генно-инженерными аналогами инсулина ультракороткого действия аспарт и лизпро. Все пациенты были обеспечены расходными материалами к ИП в полном объеме в рамках городской программы «Сахарный диабет и профилактика его осложнений».

\section{Основные результаты исследования}

Значимых изменений динамики $\mathrm{HbA}_{1 c}$ у детей и подростков, которые находились на НПИИ 3 года и более, не выявлено (среднее значение $\mathrm{HbA}_{1 c}$ до перехода

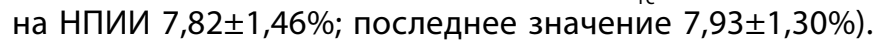
Количество пациентов с $\mathrm{HbA}_{1 c}<7,5 \%$ составило лишь $42 \%(n=100)$. Также не выявлено значимых изменений динамики $\mathrm{HbA}_{1 c}$ в зависимости от пола (у девочек $\mathrm{HbA}_{1 c}$

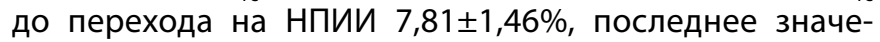
ние $\mathrm{HbA}_{1 c} 7,89 \pm 1,28 \%$; у мальчиков $\mathrm{HbA}_{1 с}$ до перехода

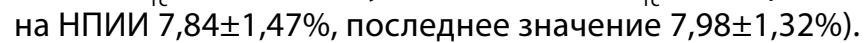

При оценке динамики $\mathrm{HbA}_{1 с}$ в разных возрастных группах значимых изменений не отмечалось. В группе 4,5-7 лет $(\mathrm{n}=18)$ отмечались лучшие показатели $\mathrm{HbA}_{1 c}$ как до перехода на НПИИ, так и учитывая его последнее значение (исходный 7,42 $0,79 \%$, последнее значение $7,21 \pm 0,69 \%)$. Однако можно отметить достоверно значимую разницу последнего значения $\mathrm{HbA}_{1 c}$ между возрастными группами - между группами 7-11 лет и 12-18 лет

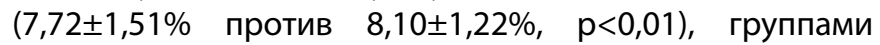
4,5-7 лет и $12-18$ лет $(7,21 \pm 0,69 \%$ против $8,10 \pm 1,22 \%$, $\mathrm{p}<0,005)$ (рис. 1).

В подгруппе детей и подростков с исходным $\mathrm{HbA}_{1 с}$ $<7,5 \%$ ( $n=107)$ отмечалось его повышение на 0,66\% (ис-

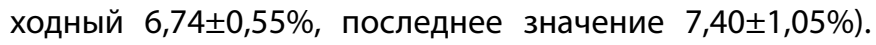
У $64 \%(\mathrm{n}=68)$ детей этой группы $\mathrm{HbA}_{1 с}$ оставался в пределах целевого значения, а у $36 \%(n=39) \mathrm{HbA}_{1 c}$ достигал уровня более $7,5 \%(p<0,001)$.

В подгруппе детей и подростков с исходным $\mathrm{HbA}_{1 c} \geq 7,5 \%(\mathrm{n}=110)$ отмечалась тенденция к снижению

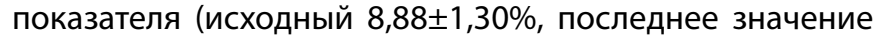
$8,46 \pm 1,32 \%)$. В этой группе лишь у $23 \%(n=25)$ человек удалось достичь $\mathrm{HbA}_{1 c}<7,5 \%$, у остальных $77 \%(\mathrm{n}=83)$ содержание $\mathrm{HbA}_{1 c}$ превышало целевой уровень, т.е. более $7,5 \%$ (р <0,001) (рис. 2, 3).

Целевые показатели $\mathrm{HbA}_{1 c}(<7,5 \%)$ обнаружены у $67 \%$ детей из группы 4,5-7 лет, у 55\% из группы 8-11 лет и 35\% из группы 12-18 лет ( $<<0,001)$.

В общей группе детей и подростков постоянно использовали ИП 169 человек (71\%). Содержание $\mathrm{HbA}_{1 с}$ в этой группе ниже на 0,6\% по сравнению с группой, В которой пациенты использовали ИП непостоянно

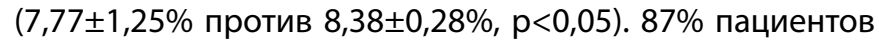
с $\mathrm{HbA}_{1 c}<7,5$ использовали ИП постоянно. В группе пациентов с $\mathrm{HbA}_{1 c} \geq 7,5 \%$ постоянно использовали ИП 69\%. Непостоянно использовали ИП 50 человек, из них большую часть, а именно 82\%, составили подростки.

В группе подростков, которые использовали ИП постоянно, содержание $\mathrm{HbA}_{1 с}$ было ниже на 0,7\% по срав- 
Медиана; ящик: Q25-Q75; усы: минимум, максимум

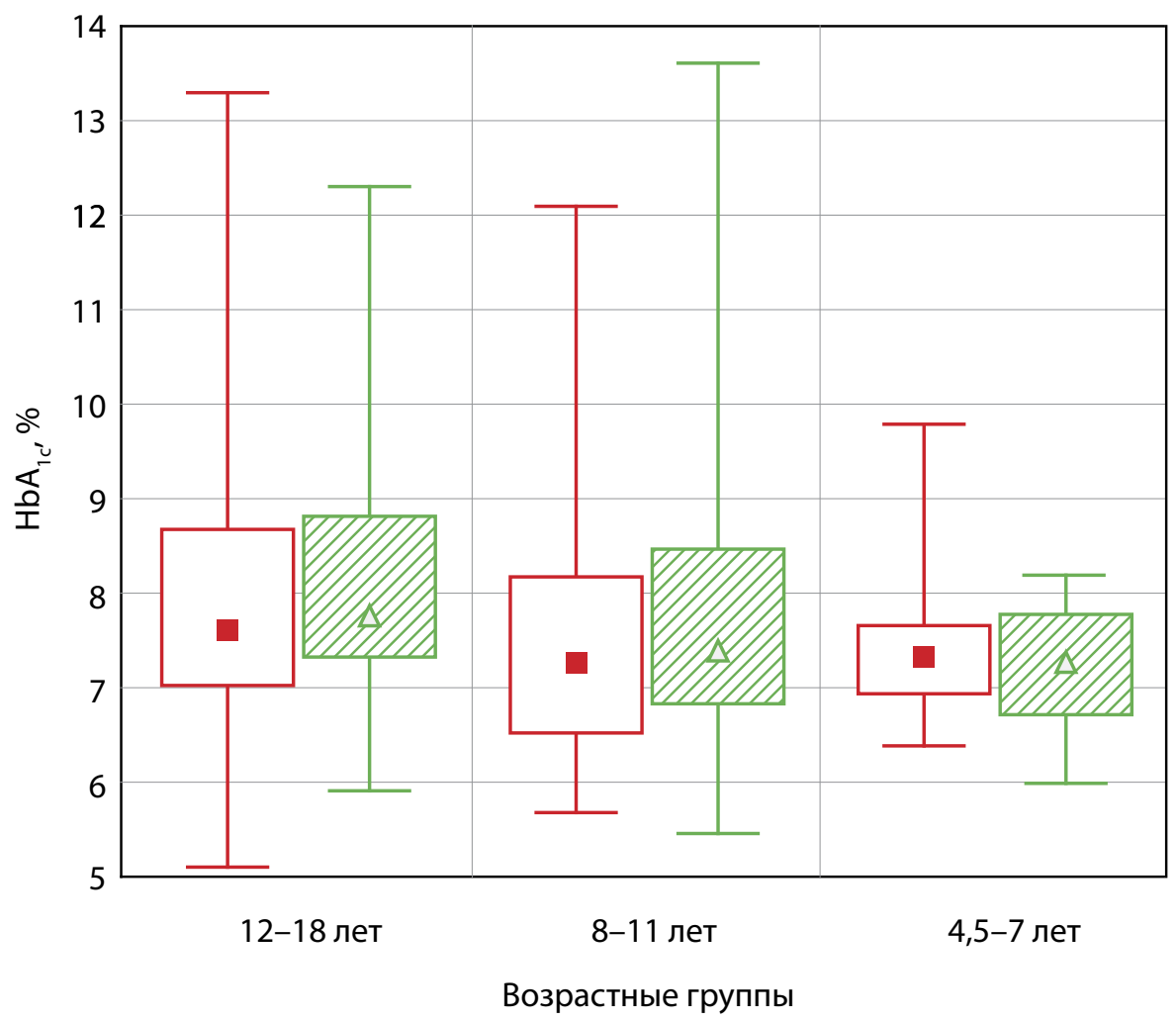

Концентрация $\mathrm{HbA}_{1 с}$ до перехода на НПИИ

$\triangle$ Последнее значение $\mathrm{HbA}_{1 c}$

Рис. 1. Динамика концентрации $\mathrm{HbA}_{1 с}$ у детей разных возрастных групп (4,5-7 лет, 8-11 лет, 12-18 лет), получающих непрерывную подкожную инфузию инсулина 3 года и более.

Медиана; ящик: Q25-Q75; усы: минимум, максимум

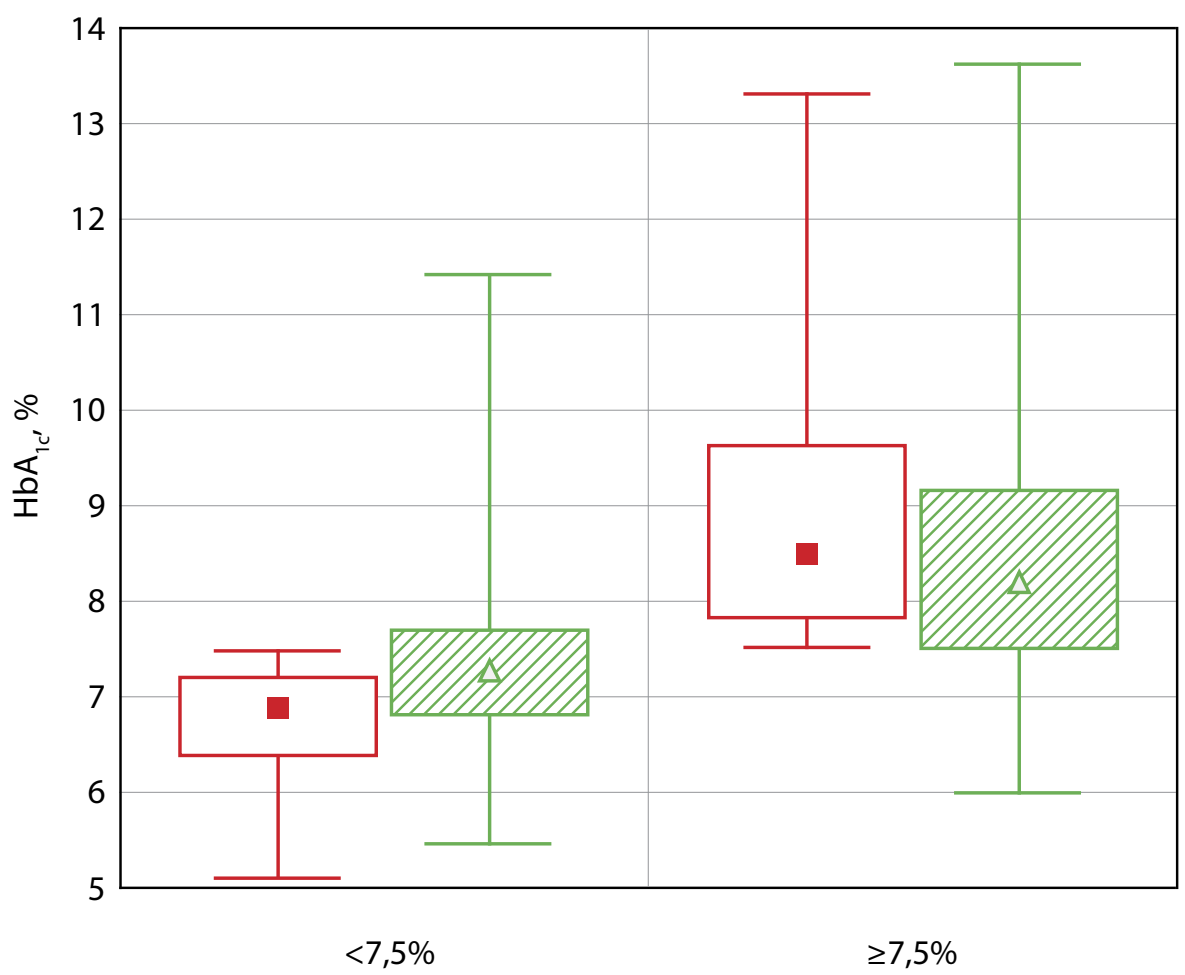

Концентрация $\mathrm{HbA}_{1 \mathrm{c}}$ до перехода на НПИИ

$\triangle$ Последнее значение $\mathrm{HbA}_{1 \mathrm{c}}$

Концентрация $\mathrm{HbA}_{1 с}$ до перехода на НПИИ

Рис. 2. Динамика концентрации $\mathrm{HbA}_{1 c}$ в зависимости от его исходного уровня. 


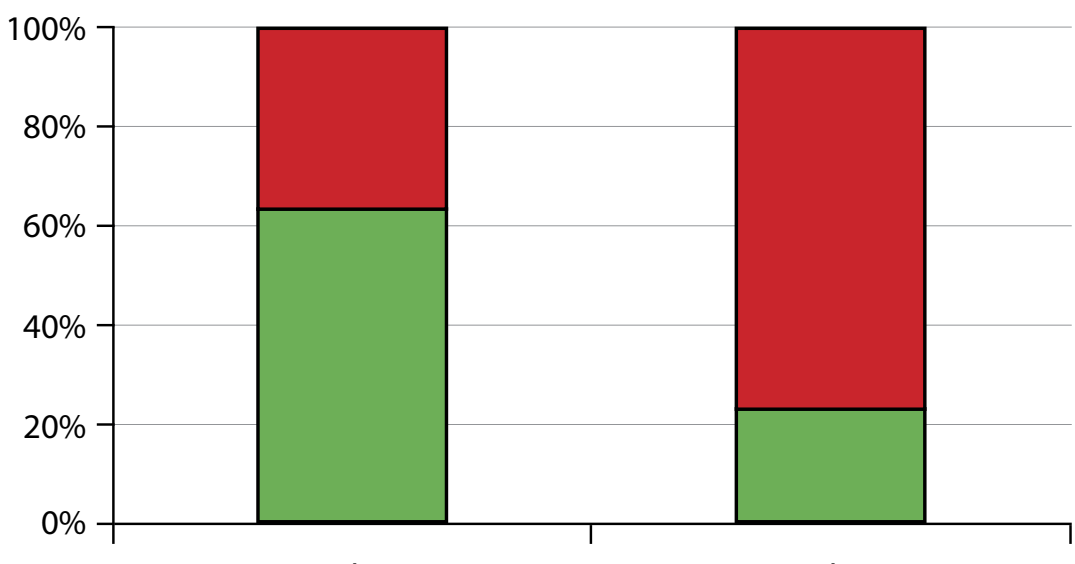

Группа с $\mathrm{HbA}_{1 \mathrm{c}}<7,5 \%$
Группа с $\mathrm{HbA}_{1 \mathrm{c}} \geq 7,5 \%$ $\square$ Количество пациентов с последним значением $\mathrm{HbA}_{1 \mathrm{c}}<7,5 \%$

Количество пациентов с последним значением $\mathrm{HbA}_{1 \mathrm{c}} \geq 7,5 \%$

Группы пациентов в зависимости от значения концентрации $\mathrm{HbA}_{1 с}$ до перехода на НПИИ

Рис. 3. Динамика изменения концентрации $\mathrm{HbA}_{1 c}$ в зависимости от его уровня до перехода на непрерывную подкожную инфузию инсулина.

нению с группой, в которой пациенты периодически

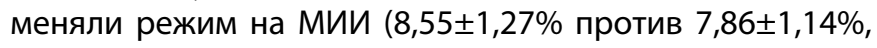
$\mathrm{p}<0,05)$. Переход пациентов на МИИ не был связан с отсутствием расходных материалов к инсулиновому дозатору, а обусловлен исключительно приверженностью пациента к тому или иному способу введения инсулина. Характеристики динамики $\mathrm{HbA}_{1 с}$ в разных возрастных группах в зависимости от постоянства использования ИП можно видеть на рис. 4.

В процессе исследования также получены данные о влиянии регулярности использования НМГ на уровень $\mathrm{HbA}_{1}$. В общей группе не использовали НМГ или использовали редко (1-2 раза в год, при госпитализации) 101 человек (42\%); использовали постоянно либо не реже 1 нед

Медиана; ящик: Q25-Q75; усы: минимум, максимум

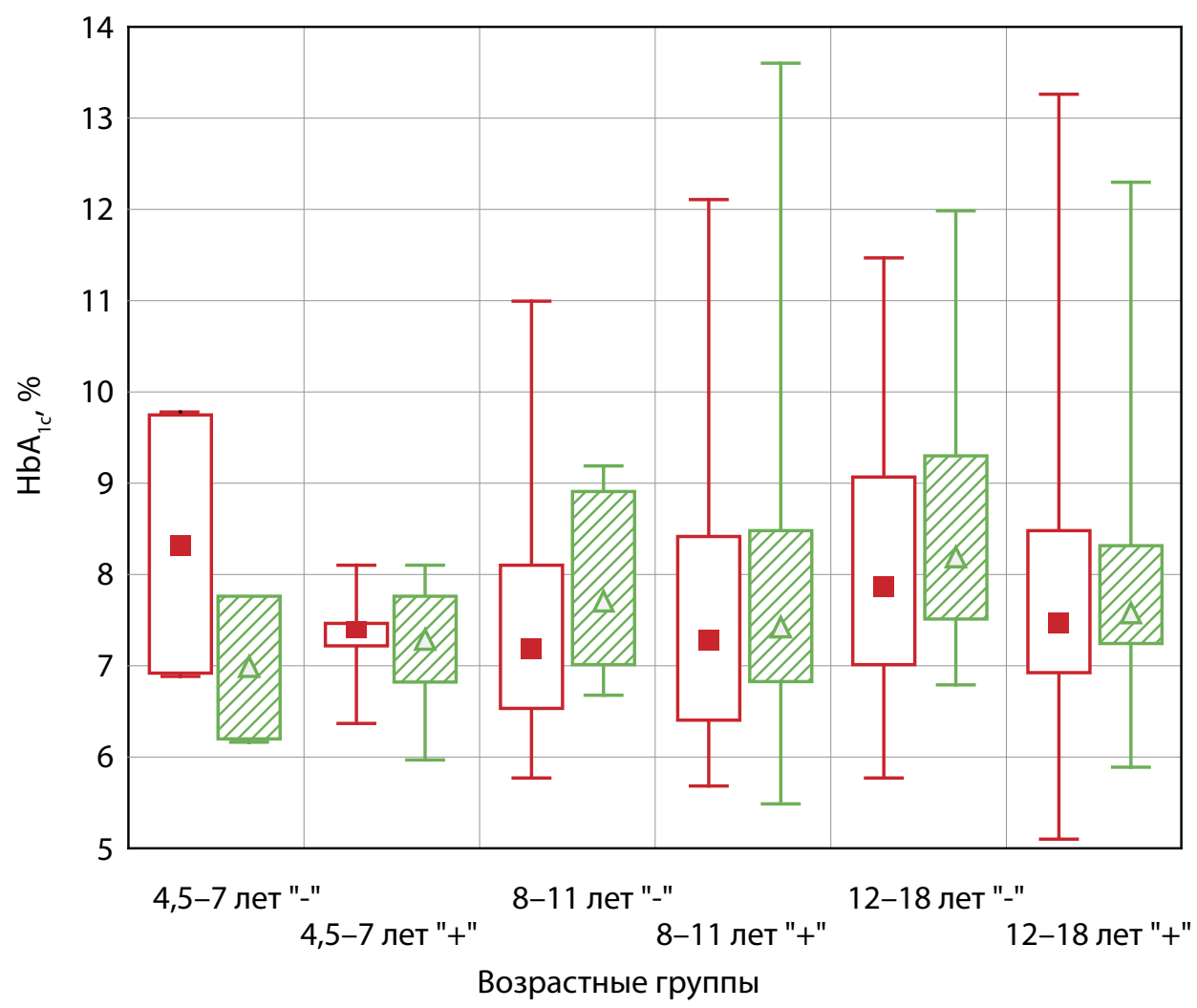

Концентрация $\mathrm{HbA}_{1}$ до перехода на НПИИ

$\Delta$ Последнее значение $\mathrm{HbA}_{1 с}$

«+» Постоянно на помповой инсулинотерапии

\section{«-» Переходят}

периодически на МММ с помощью шприц-ручки

Рис. 4. Динамика концентрации $\mathrm{HbA}_{1 с}$ в разных возрастных группах в зависимости от постоянства использования инсулиновой помпы. 
Медиана; ящик: 25\%, 75\%; усы: минимум, максимум

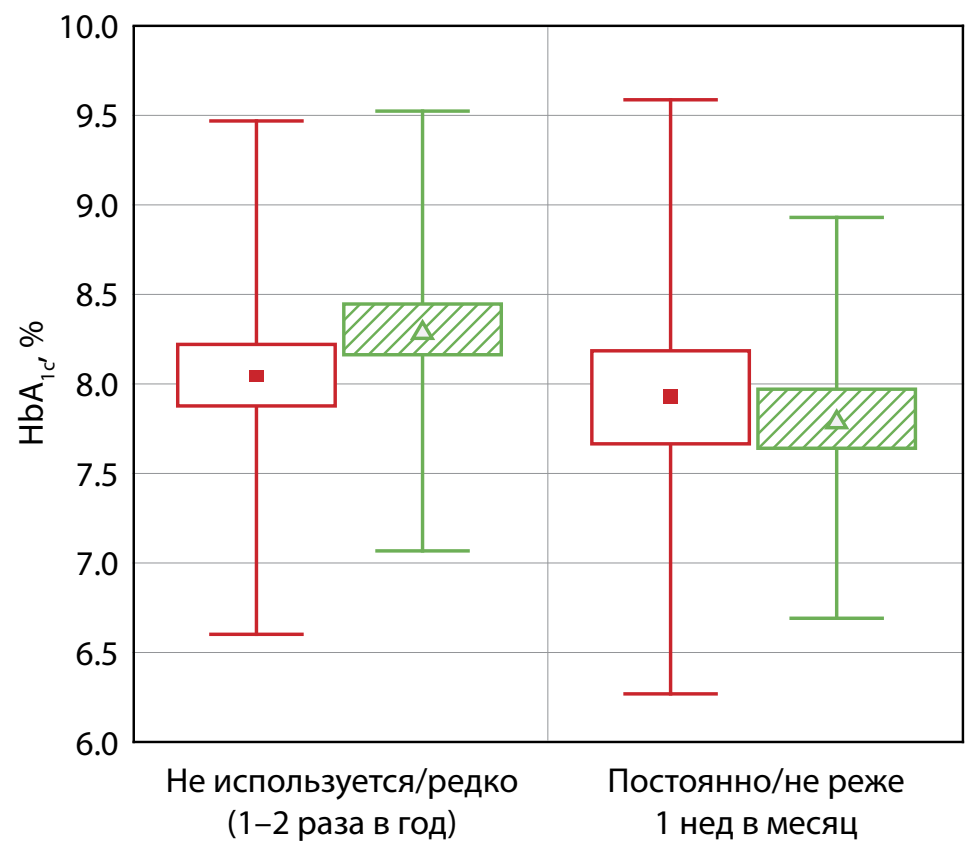

Концентрация $\mathrm{HbA}_{1 \mathrm{c}}$ до перехода на НПИИ

$\triangle$ Последнее значение $\mathrm{HbA}_{1 \mathrm{c}}$

Частота использования НМП

Рис. 5. Динамика концентрации $\mathrm{HbA}_{1 с}$ в возрастной группе 12-18 лет в зависимости от частоты непрерывного мониторирования глюкозы.

в месяц 70 человек (29\%), из них 40 подростков. У детей и подростков, использовавших НМГ не реже 1 нед в месяц, имелась тенденция к более низким значениям концентрации $\mathrm{HbA}_{1 с}$ по сравнению с детьми и подростками, которые не использовали НМГ либо использовали редко, однако полученные показатели не достигли статисти-

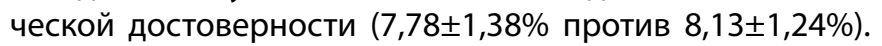
Достоверно ниже был $\mathrm{HbA}_{1 с}$ у детей в группе 12-18 лет, использовавших НМГ постоянно, по сравнению с груп-

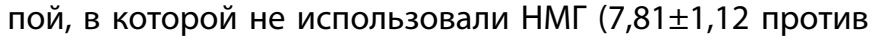
$8,3 \pm 1,23 \%, p<0,05)$ (рис. 5).

В общей группе пациентов КБ использовали 71\% пациентов, но статистически значимых изменений $\mathrm{HbA}_{1 с}$ в группах пациентов, которые использовали и не использовали КБ, не выявлено. Также не выявлено статистически значимых изменений $\mathrm{HbA}_{1 с}$ при использовании КБ в зависимости от пола и возраста.

\section{Дополнительные результаты исследования}

В анкетах пациенты ответили на вопрос о том, по каким причинам они временно переходят с ППИИ на шприц-ручки. Большинство ответивших, а именно 82\%, переходят с ППИИ на МИИ в летний период. Меньшее количество детей переходят на МИИ в период болезни, занятий спортом и в связи с появлением липодистрофий в местах инъекций инсулина (табл. 1). Пациенты, не использующие НМГ, также ответили на вопрос о причинах, по которым они не используют НМГ. $44 \%$ опрошенных выделяют отсутствие финансовой возможности как основную причину, по которой они не используют НМГ. Также пациенты не использовали НМГ в связи с отсутствием технической возможности ИП, отсутствием желания и неудобством использования устройства (табл. 2).

Таблица 1. Причины временного перехода с помповой инсулинотерапии на режим множественных инъекций инсулина

\section{Причины временного перехода с нПИИ на шприц-ручки}

Летний период

Занятия спортом

Период болезни

\section{Кол-во человек, n (\%); всего 60 ответивших пациентов}

49 (82)

$6(10)$

Липодистрофии

Таблица 2. Причины отказа от непрерывного мониторирования глюкозы

\begin{tabular}{lc}
\hline \multicolumn{1}{c}{ Причины отказа от НМг } & $\begin{array}{c}\text { Кол-во человек, } \\
\text { n (\%); всего } \\
\text { 46 ответивших } \\
\text { пациентов }\end{array}$ \\
\hline Нет финансовой возможности & $20(44)$ \\
\hline Нет технической возможности & $8(17)$ \\
Нет желания & $8(17)$ \\
\hline Неудобно & $4(9)$ \\
Не предлагали & $2(9)$ \\
Неточные показания & $2(4)$ \\
\hline Аллергия на пластырь & $2(4)$ \\
\hline
\end{tabular}




\section{ОБСУЖДЕНИЕ}

Применение дозаторов инсулина длительностью не менее 3 лет при лечении СД1 у детей и подростков позволяет сохранять метаболическую компенсацию заболевания. Однако к значимому улучшению показателя хронической гипергликемии помповая инсулинотерапия не привела. В группе 239 пациентов с СД1 отмечалось повышение последнего значения $\mathrm{HbA}_{1 c}$ на $0,1 \%$ от исходного, и его уровень составлял $7,93 \pm 1,3 \%$, что свидетельствовало о недостаточной долгосрочной эффективности терапии СД методом НПИИ. Полученный результат сопоставим с данными международных исследований. Семилетнее ретроспективное международное многоцентровое исследование в трех странах продемонстрировало отсутствие значимого улучшения гликемического контроля у детей, получающих инсулин с помощью инсулинового дозатора, показатель $\mathrm{HbA}_{1 с}$ снизился лишь на 0,04\% [7]. Вместе с тем следует обратить внимание на неочевидные, но важные детали при анализе динамики показателей хронической гипергликемии при длительности использования НПИИ более 3 лет в контексте клинической характеристики включенных в настоящее исследование пациентов. Так, уровень $\mathrm{HbA}_{1 c}$ на момент исследования составил 7,93\%, а число пациентов, достигших уровня $\mathrm{HbA}_{1 c}$ менее 7,5\%, - 42\%. В то время как, согласно данным Федерального регистра СД (Дедов И.И. и соавт., 2017), показатели $\mathrm{HbA}_{1 c}$ менее 7,5\% отмечаются лишь у $32,2 \%$ детей и 25,5\% подростков. У пациентов с исходным уровнем $\mathrm{HbA}_{1 с}$ более 7,5\% отмечалась тенденция к снижению показателя. Некоторое повышение значений $\mathrm{HbA}_{1 c}$ у пациентов с исходным уровнем $\mathrm{HbA}_{1 c}$ менее 7,5\% (на 0,66\%) через 3 года лечения может отчасти отражать процесс естественного течения заболевания в случаях перевода на НПИИ при длительности заболевания менее двух лет. Указанные факты свидетельствуют в пользу бесспорной эффективности НПИИ. Однако применение любого вновь предложенного метода лечения в реальной клинической практике сопровождается отличительными особенностями от результатов лечения в рамках контролируемых исследований. Изучение факторов, влияющих на эффективность помповой инсулинотерапии, в ряде исследований показало зависимость результатов лечения от возраста пациента. С возрастом ухудшаются показатели метаболического контроля вне зависимости от длительности заболевания $[12,16]$. В настоящем исследовании лучшие показатели наблюдаются в возрастной группе 4,5-7 лет, где количество пациентов с $\mathrm{HbA}_{1 c}<7,5 \%$ составляет $67 \%$, в то время как в группе детей 8-11 лет 54\% детей с целевым уровнем $\mathrm{HbA}_{1 c}$, а в группе подростков (12-18 лет) лишь $35 \%$ человек имеют целевой уровень $\mathrm{HbA}_{1 c}$. Ухудшение гликемического контроля у подростков может быть обусловлено как гормонально-метаболическими изменениями в процессе полового развития, так и психологическими особенностями этого периода, стремлением стать независимыми и свободными от родителей. Также тенденция к повышению уровня $\mathrm{HbA}_{1 c}$ с возрастом, возможно, связана с участием родителей в управлении СД у маленьких детей. В ряде исследований было отмечено, что фактор приверженности лечению явля- ется значимым в достижении оптимальных результатов. Так, целевые показатели $\mathrm{HbA}_{1 с}$ у комплаентных пациентов до перехода на НПИИ сохраняли свои значения и на протяжении длительного времени использования НПИИ $[16,17]$. Аналогичная тенденция прослеживалась В настоящем исследовании. Пациенты с целевым уровнем $\mathrm{HbA}_{1 c}(<7,5 \%)$ до перехода на НПИИ имели его последнее значение также в пределах целевого, а в группе пациентов с исходным уровнем $\mathrm{HbA}_{1 с} \geq 7,5 \%$ лишь $23 \%$ достигли целевых значений. Это может указывать на определяющую роль фактора приверженности пациентов и их родителей к лечению СД с помощью инсулиновых дозаторов. В некоторых исследованиях лучший гликемический контроль отмечался у мальчиков [7], однако в настоящем исследовании не обнаружено значимых изменений $\mathrm{HbA}_{1 с}$ в зависимости от пола пациентов.

В настоящее время широко обсуждается роль методов НМГ в поддержании стойкой метаболической компенсации при помповой инсулинотерапии. Убедительно подтверждена связь лучших показателей гликемии при использовании НМГ [18]. Дискутабельным остается вопрос приоритетов и необходимости использования технически более трудоемких методов метаболического контроля. Однако результат стойкой гликемии у пациентов при использовании НМГ в нашем исследовании свидетельствует в пользу его применения. Исследование продемонстрировало, что терапия эффективнее у пациентов, использующих НМГ не реже 1 нед в месяц в сравнении с пациентами, которые вообще не использовали или использовали редко НМГ. Также представляется все больше доказательств того, что использование КБ может улучшать метаболический контроль и удовлетворенность лечением. Использование КБ в настоящем исследовании не продемонстрировало положительного действия на метаболический контроль. Вопрос о частой смене режимов инсулинотерапии (периодический переход с помповой терапии на МИИ) в последнее время становится все более актуальным. Применение различных методов введения инсулина в зависимости от условий жизни дает не только свободу выбора в лечении СД, но и позволяет гибко управлять заболеванием. Однако зачастую это приводит к ухудшению метаболического контроля. Анализ показателей компенсации заболевания в группе пациентов с постоянным применением НПИИ показал лучшие результаты в сравнении с пациентами, которые периодически переходили на МИИ с помощью шприц-ручек.

\section{ЗАКЛЮЧЕНИЕ}

В результате проведенного исследования не выявлено статистически значимого изменения показателей $\mathrm{HbA}_{1 с}$ у детей и подростков на НПИИ длительностью 3 года и более в сравнении с исходным значением. Вместе с тем длительное поддержание метаболической компенсации заболевания при использовании НПИИ свидетельствует в пользу эффективности данного метода лечения. Среднее значение $\mathrm{HbA}_{1 c}$ у детей и подростков, находящихся на НПИИ 3 года и более, составляло 7,93\%, а количество пациентов с концентрацией $\mathrm{HbA}_{1 c}$ $<7,5 \%$ насчитывало 42\%, что может свидетельствовать о необходимости регулярного терапевтического обу- 
чения пациентов и их родителей методам управления заболеванием. В группе пациентов, использующих ИП постоянно, отмечается лучший гликемический контроль, в сравнении с группой, в которой пациенты периодически переходили с НПИИ на МИИ с помощью шприц-ручки. При этом следует отметить, что переход осуществлялся при полной доступности к расходным материалам. Также полученные данные указывают на то, что использование НМГ не реже 1 недели в месяц положительно влияет на величину $\mathrm{HbA}_{1 c^{\prime}}$ а использование КБ не повлияло на улучшение метаболического контроля.

\section{ДОПОЛНИТЕЛЬНАЯ ИНФОРМАЦИЯ}

Финансирование работы. Исследование проведено в рамках бюджетного финансирования учреждения.

Конфликт интересов. Авторы заявляют об отсутствии конфликта интересов.

Участие авторов:Башнина Е.Б.- концепция идизайн исследования, редактирование, финальное утверждение рукописи; Царгасова И.М. обработка и анализ полученных данных, написание текста; Клиценко О.А., Царгасова И.М. - статистическая обработка материала. Все авторы внесли существенный вклад в проведение исследования и подготовку статьи, прочли и одобрили до публикации.

\section{СПИСОК ЛИТЕРАТУРЫ | REFERENCES}

1. Эндокринные заболевания у детей и подростков: руководство для врачей / Под ред. Е.Б. Башниной. - М.: ГЭОТАР-Медиа, 2017. C. 26-41. [Endokrinnyye zabolevaniya u detey i podrostkov: rukovodstvo dlya vrachey. Ed. by E.B. Bashnina. Moscow: GEOTAR-Media; 2017. p. 26-41. (In Russ.)]

2. Башнина Е.Б., Трясова Т.Ю., Корналевская А.О. Инсулинотерапия у подростков с сахарным диабетом: проблемы и пути решения // Лечащий врач. - 2012. - №6. - C. 40. [Bashnina EB, Tryasova TYu, Kornalevskaya AO. Insulinoterapiya u podrostkov s sakharnym diabetom: problemy i puti resheniya. Lechashchii vrach. 2012;(6):26-28. (In Russ.)]

3. Капланян М.В., Ворохобина Н.В., Ряснянский В.Ю. Предикторы диабетической нефропатии у больных сахарным диабетом 1 типа. / Достижения персонализированной медицины сегодня - результат практического здравоохранения завтра: сб. тез. VII Всероссийского конгресса эндокринологов; Москва, 2016, 2-5 марта. - М.: УП Принт, 2016. [Kaplanyan MV, Vorokhobina NV, Ryasnyanskiy Vu. Prediktory diabeticheskoy nefropatii u bol'nykh sakharnym diabetom 1 tipa. (Congress proceedings) Dostizheniya personalizirovannoy meditsiny segodnya — rezul'tat prakticheskogo zdravookhraneniya zavtra; Moscow, 2016 March 2-5. Moscow: UP Print; 2016. (In Russ.)]

4. Karges B, Schwandt A, Heidtmann B, et al. Association of insulin pump therapy with severe hypoglycemia, ketoacidosis, and glycemic control among children, adolescents and young adult with type 1 diabetes. JAMA. 2017;318(14):1358-1366. doi: https://doi.org/10.1001/jama.2017.13994

5. Majedah M. AbdulRasoul, Mousa M, Al-Mahdi M, et al. A comparison of continuous subcutaneous insulin infusion vs. multiple daily insulin injection in children with type i diabetes in Kuwait: glycemic control, insulin requirement, and BMI. Oman Med J. 2015;30(5):336-343. doi: https://doi.org/10.5001/omj.2015.69

6. Batajoo RJ, Messina CR, Wilson TA. Long-term efficacy of insulin pump therapy in children with type 1 diabetes mellitus. J Clin Res Pediatr Endocrinol. 2012;4(3):127-131. doi: https://doi.org/10.4274/Jcrpe.751

7. Mameli C, Scaramuzza AE, Ho J, et al. A 7-year follow-up retrospective, international, multicenter study of insulin pump therapy in children and adolescents with type 1 diabetes. Acta Diabetol. 2014;51(2):205-210. doi: https://doi.org/10.1007/s00592-013-0481-y

8. Langendam M, LuijfYM, Hooft L, et al. Continuous glucose monitoring systems for type 1 diabetes mellitus. Cochrane Database Syst Rev. 2012;1:CD008101. doi: https://doi.org/10.1002/14651858.CD008101.pub2

9. Burden AC. ACP Journal Club. Review: continuous glucose monitoring reduces $\mathrm{HbA} 1 \mathrm{C}$ more than self-monitoring in type 1 diabetes. Ann Intern Med. 2013;158(6):JC9. doi: https://doi.org/10.7326/0003-4819-158-6-201303190-02009
10. Cukierman-Yaffe T, Konvalina N, Cohen O. Key elements for successful intensive insulin pump therapy in individuals with type 1 diabetes. Diabetes Res Clin Pract. 2011;92(1):69-73. doi: https://doi.org/10.1016/j.diabres.2011.01.002

11. American Diabetes Association. 12. Children and adolescents: standards of medical care in diabetes-2018. Diabetes Care. 2018;41(Suppl 1):S126-S136. doi: https://doi.org/10.2337/dc18-S012

12. Campbell MS, Schatz DA, Chen V, et al.;T1D Exchange Clinic Network. A contrast between children and adolescents with excellent and poor control:T1D Exchange clinic registry experience. Pediatr Diabetes. 2014;15(2):110-117. doi: https://doi.org/10.1111/pedi.12067

13. Шестакова М.В., Майоров А.Ю., Филиппов Ю.И., и др. Федеральные клинические рекомендации по помповой инсулинотерапии и непрерывному мониторированию гликемии у больных сахарным диабетом. Проект // Проблемы эндокринологии. 2015. — T.61. — №6. - C. 55-78. [Shestakova MV, Mayorov AYu, Philippov Yul, et al. Russian national guidelines on insulin pump therapy and continuous glucose monitoring for diabetes mellitus patients. DRAFT. Problemy endokrinologii. 2015;61(6):55-78. (In Russ.)]. doi: https://doi.org/10.14341/probl201561655-78

14. Pickup JC, Sutton AJ. Severe hypoglycaemia and glycaemic control in type 1 diabetes: meta-analysis of multiple daily insulin injections compared with continuous subcutaneous insulin infusion. Diabet Med. 2008;25(7):765-774. doi: https://doi.org/10.1111/j.1464-5491.2008.02486

15. Петеркова В.А., Кураева Т.Л., Емельянов А.О., и др. Помповая инсулинотерапия у детей и подростков. Методическое пособие для педиатров-эндокринологов. - М.: Эндокринологический научный центр, 2011. - 48 с. [Peterkova VA, Kuraeva TL, Emel'yanov $A O$, et al. Pompovaya insulinoterapiya u detey i podrostkov. Metodicheskoe posobie dlya pediatrov-endokrinologov. Moscow: Endocrinological research center; 2011.48 p. (In Russ.)]

16. Wood JR, Miller KM, Maahs DM, et al. Most youth with type 1 diabetes in the T1D Exchange Clinic Registry do not meet American Diabetes Association or International Society for Pediatric and Adolescent Diabetes clinical guidelines. Diabetes Care. 2013;36(7):2035-2037. doi: https://doi.org/10.2337/dc12-1959

17. Blackman SM, Raghinaru D, Adi S, et al. Insulin pump use in young children in the T1D Exchange clinic registry is associated with lower hemoglobin A1c levels than injection therapy. Pediatr Diabetes. 2014;15(8):564-572. doi: https://doi.org/10.1111/pedi.12121

18. Sheikh K, Bartz SK, Lyons SK, DeSalvo DJ. Diabetes device use and glycemic contol among youth with type 1 diabetes: a SingleCenter, Cross-Sectional Study. J Diabetes Res. 2018;2018:5162162. doi: https://doi.org/10.1155/2018/5162162

\section{ИНФОРМАЦИЯ ОБ АВТОРАХ [AUTHORS INFO]}

*Башнина Елена Борисовна д.м.н., профессор [Elena B. Bashnina, MD, PhD, Professor]; адpec: 191015, Россия, Санкт-Петербург, ул. Кирочная, д. 41 [address: 41 Kirochnaya ul., 191015 Saint-Petersburg, Russia,]; ORCID: http://orcid.org/0000-0002-7063-1161; eLibrary SPIN: 5568-0690; e-mail: bashnina@mail.ru

Царгасова Ирина Михайловна [Irina M. Tsargasova, MD]; ORCID: https://orcid.org/0000-0002-4487-4282; eLibrary SPIN: 9674-6001; e-mail: tsargasova_i@mail.ru

Клиценко Ольга Анатольевна к.б.н., доцент [Olga A. Klitsenko, PhD in Biology, associate professor]; ORCID: https://orcid.org/0000-0002-2686-8786; eLibrary SPIN: 7354-3080; e-mail: olkl@yandex.ru 


\section{ЦИТИРОВАТЬ:}

Башнина Е.Б., Царгасова И.М., Клиценко О.А. Факторы, ассоциированные с метаболической компенсацией у детей и подростков с сахарным диабетом 1 типа при применении систем непрерывного подкожного введения инсулина // Сахарный диабет. — 2019. — T. 22. — №6. — C. 550-558. doi: https://doi.org/10.14341/DM10208

\section{TO CITE THIS ARTICLE:}

Bashnina EB, Tsargasova IM, Klitsenko OA. Systems of continuous subcutaneous insulin infusion to maintain metabolic compensation for type 1 diabetes mellitus among children and adolescents. Diabetes Mellitus. 2019;22(6):550-558. doi: https://doi.org/10.14341/DM10208 